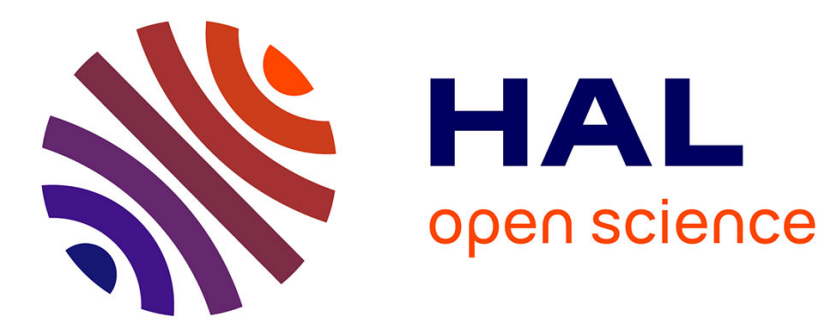

\title{
Fracture spacing in tensile brittle layers adhering to a rigid substrate
}

Veronique Lazarus

\section{To cite this version:}

Veronique Lazarus. Fracture spacing in tensile brittle layers adhering to a rigid substrate. EPL Europhysics Letters, 2017, 117 (2), pp.24002. 10.1209/0295-5075/117/24002 . hal-01904342

\section{HAL Id: hal-01904342 \\ https://hal.science/hal-01904342}

Submitted on 29 Oct 2018

HAL is a multi-disciplinary open access archive for the deposit and dissemination of scientific research documents, whether they are published or not. The documents may come from teaching and research institutions in France or abroad, or from public or private research centers.
L'archive ouverte pluridisciplinaire HAL, est destinée au dépôt et à la diffusion de documents scientifiques de niveau recherche, publiés ou non, émanant des établissements d'enseignement et de recherche français ou étrangers, des laboratoires publics ou privés. 


\title{
Fracture spacing in tensile brittle layers adhering to a rigid sub- strate
}

\author{
VÉRONIQUE LAZARUS \\ Laboratoire FAST, Univ Paris Sud, CNRS, Université Paris-Saclay, F-91405, Orsay, France
}

PACS 46.50.+a - Fracture mechanics, fatigue and cracks

PACS 62.20.M- - Structural failure of materials

PACS 89.75.Kd - Patterns

\begin{abstract}
A natural question arising when observing crack networks in brittle layers as e.g. paints, muds, skins, pottery glazes, coatings, ceramics is what determines the distance between cracks. This apparently simple question received a wealth of more or less complex and appropriate answers, but no consensus has emerged. Here, we show that the cracks interact mutually as soon as the spacing between them is smaller than ten times the thickness of the layer. Then, a simple Griffith-type balance between the elastic deformation energy and the fracture bulk and debonding costs captures a broad number of observations, going from the square-root or linear increase of the spacing with the thickness, to its decrease with loading until saturation. The adhesion strength is identified as playing a key role in these behaviour changes. As illustration, we show how the model can be applied to study the influence of the layer thickness on crack patterns. We believe that the versatility of the approach should permit wide applicability, from geosciences to engineering.
\end{abstract}

(a)

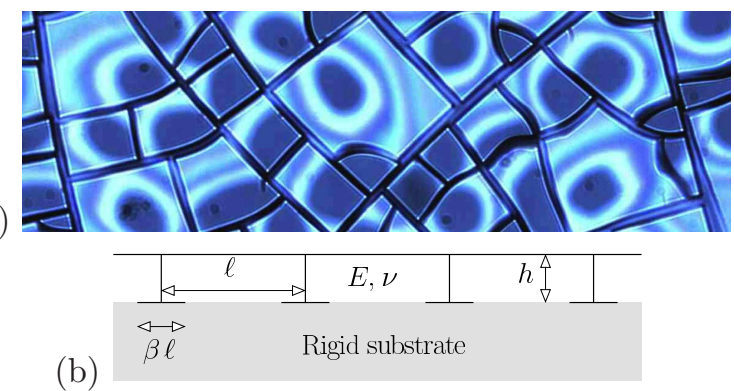

Fig. 1: (a) Drying of a colloidal film on a rigid substrate [1] (courtesy L. Pauchard). The channeling cracks divide the transparent layer into polygonal cells provided that $h>h_{c} \sim$ $10 \mu \mathrm{m}$. Debonding from the substrate appears if $h>h_{d} \sim$ $30 \mu \mathrm{m}$. The picture (width $\sim 600 \mu \mathrm{m}$ ) corresponds to $h \simeq 35$ $\mu \mathrm{m}$. Delamination is evidenced by the interference fringes. (b) Cross-section sketch of the layer with notations.

Crack networks invading a tensile brittle layer can be contemplated in our daily lives, for instance in dishes, glazes of fine pottery, road pavements, skins during cold weather, muds [2], paintings [3]. Such patterns are common on Earth $[4,5]$ and other planets $[6,7]$ within a wide range of scales. The cracks represent a significant hurdle in manufactured coatings (protective films, composites, MEMS, thermal barriers, electrodes of Li-Ion Bat- teries ...) [8] where tensile stresses are induced by deposition processes (e.g. epitaxy, drying of suspensions [2,9]) or in-service loading conditions (e.g., thermal expansion misfit [10]). Conversely, their control and harness have been pointed out to be a promising cutting method in micro-fabrication technologies [11]. The typical spacing between cracks, whether they form a polygonal pattern (Fig. 1a) or a parallel array, generally increases with the layer thickness and decreases with the loading until saturation. These two features have been evidenced in experiments [1,12-16] and in layered rocks [17]. These observations yield the development of different models, which fail to reach a consensus [18].

The final crack pattern results from the nucleation and subsequent propagation of the cracks. Most of the models can be categorised into two classes, focusing either on (i) the nucleation or on (ii) the propagation phase. Nucleation is commonly considered to occur only if and where the tensile stress is above a given threshold. The spacing is then predicted to be linear with the thickness [19] and the saturation is explained by the existence, when the spacing is small enough, of a compressive stress in the center of one cracked segment, prohibiting the creation of a new crack $[17,20-23]$. But the choice of the segment center as nucleation point is arbitrary, the stresses remain ten- 
sile elsewhere; a crack may nucleate there and modify the local stress state changing it from compressive to tensile, allowing the crack to propagate down to the substrate [24]. More generally, this first approach omits the fundamental property of cracks to change the local stress state and to concentrate the loading at their tip [25]. A second type of models focus on the propagation of preexisting cracks by using Griffith's energy type arguments [22,26]: cracks propagate by balancing the elastic energy released during propagation with the formation costs of the fracture surface. But up to now, this approach has failed to predict the saturation and the linear trend observed in most experiments $[1,12-14,16]$. The first class of models has then be invoked to palliate this apparent inadequacy [22].

In this Letter, we show how Griffith's type arguments alone can capture most of the above observations with a minimum set of physically well-based parameters. We consider a brittle layer in tension adhering to a rigid substrate and two types of fracture patterns : channeling cracks through the layer and debonding from the substrate (Fig. 1b). We obtain the spacing between channeling cracks by balancing the elastic energy stored in the deformed layer with the energy cost to be paid to create fracture surfaces in the layer or to debond surfaces from the substrate. First, we retrieve the existence of a stress or thickness level below which no crack can channel through the layer, since the elastic energy is insufficient to overcome the fracture costs $[1,26]$. Above, we find two regimes. First, for low enough stress levels, channeling occurs without debonding and a complex dependence of the spacing with the loading is found: Cracks first appear and channel through the layer at places initiated by some local weaknesses until they start to interact with each others. The spacing is then fixed by the interaction between cracks. This transition from a disordered to a deterministic behaviour [27] occurs for quite large values of the spacing ( $\simeq 10$ times the film thickness). Second, for higher stress levels, the layer debonds progressively at the expense of channeling, so that the spacing between cracks saturates. In this regime, the spacing scales linearly with the thickness and the slope depends on the sole ratio between bulk and interface fracture energies. We quantify all these aspects through dimensionless scaling relations and use them to understand the thickness dependence of the crack patterns.

We consider a homogeneous brittle layer of thickness $h$, Young's modulus $E$ and Poisson's ratio $\nu$ (Fig. 1b), under plane strain condition $^{1}$, adhering perfectly to a rigid substrate, and loaded by an increasing uniform isotropic tensile prestress $\sigma_{0}\left(\sigma_{0}>0\right)$ or equivalent misfit strain $\varepsilon_{0}=-\frac{1-2 \nu}{E} \sigma_{0}$ (which may originate for instance, from drying [29] or thermal shrinkage). In the absence of any crack, the layer is under biaxial tension ${ }^{2} \sigma=\frac{1-2 \nu}{1-\nu} \sigma_{0}$.

\footnotetext{
${ }^{1}$ plane stress condition and extension to $3 \mathrm{D}$ are discussed in supplemental material [28]; Only the main results are given in the main part of the manuscript.

${ }^{2}$ Notice that numerous papers (for instance [26,30,31]), consider
}

The fracture costs (per unit length in the direction normal to the Fig. 1b) associated with channeling are $N \Gamma_{b} h$ in the layer, and those associated with debonding along the interface are $N L \Gamma_{i}$, where $N$ is the number of channeling cracks and $L$ the length of the debonding zones within one segment. $\Gamma_{b}$ and $\Gamma_{i}$ denote the energies needed to create one unit crack area in the bulk of the layer and along the interface respectively.

We assume that the final crack pattern corresponds to the global minimum of the total energy, defined as the sum of the bulk elastic and both fracture energies $[32,33]$. This strong hypothesis leads to quite simple and intelligible results. It has been shown to be realistic in some experiments (e.g., [34-36]), but could only be validated scrupulously by solving incrementally the real evolution problem from the initiation of the cracks (which is highly material and heterogeneities dependent) to the propagation of their tips. This task is, with a few exceptions [37], out of reach. Intuitively, one can conjecture that this minimum energy state is attainable provided that the cracks are not trapped in a local minimum (e.g. that enough defects are present to nucleate cracks $[38,39])$ and that the system has enough latitude to reach this state. In this simplified view, the channeling cracks are characterised by their final spacing alone so that any attempt to study the time evolution of these fractures, whether formed simultaneous or incremental, is not relevant.

Using a 1D thin film approximation of this problem [31], this global minimum has been shown [40] to correspond to a periodic crack pattern partitioning the layer into segments of equal length $\ell$, the edges of which subsequently debond from the substrate over a length $\beta \ell(0 \leq \beta \leq 1)$. But this 1D approach cannot take into account any strain gradient in the thickness of the layer. This may be questionable once the spacing between cracks becomes too small in comparison to the thickness, to consider the segment between two cracks as a 1D film. Moreover, this model involves some additional bonding layer between the substrate and the layer, which renders any quantitative comparison with experiments, as yet, unreachable [41]. Also, we aim here to generalise the results by avoiding the introduction of any auxiliary bonding layer and by taking into account stress variations in the layer thickness. We are looking for the crack $(\alpha \equiv h / \ell)$, and debonding $(\beta)$ densities, that minimises the total energy. For a given value of $\nu$, this energy can be written under dimensionless form:

$$
\mathcal{E}_{\mathrm{tot}}=\alpha U(\alpha, \beta)+\frac{\alpha}{\Sigma_{b}^{2}}+\frac{\beta}{\Sigma_{i}^{2}}
$$

where the dimensionless elastic energy of one segment $U(\alpha, \beta)$ is defined for $\sigma_{0}=1, h=1, \ell=1 / \alpha, E=1$. The dimensionless load parameters $\Sigma_{b}$ and $\Sigma_{i}$ (where the subscripts $b$ and $i$ stand for bulk and interface, respec-

$\sigma$ as the loading parameter instead of $\sigma_{0}$. This is suitable for thin film approximations, but renders any systematic $2 \mathrm{D}$ or $3 \mathrm{D}$ study somewhat ambiguous. 
tively) are defined by

$$
\Sigma_{b}=\sigma_{0} \sqrt{\frac{h}{\Gamma_{b} E}} \text { and } \Sigma_{i}=\frac{\Sigma_{b}}{\sqrt{\gamma}}, \text { with } \gamma=\Gamma_{i} / \Gamma_{b} .
$$

Those terms quantify the balance between the elastic and fracture energies for the bulk and the interface respectively. This minimisation yields $\alpha$ as a function of two dimensionless parameters, namely $\Sigma_{b}$ and $\gamma$. We assume that the channeling cracks do not propagate simultaneously with debonding ${ }^{3}$, so that we can proceed in three independent steps: (i) consider the case without debonding and determine the critical loading level $\Sigma_{b}^{c}$ necessary to obtain a given channeling crack density $\alpha$; (ii) look for the threshold $\Sigma_{i}^{c}$ corresponding to debonding for a given value of $\alpha$; (iii) balance $\Sigma_{b}^{c}$ with $\Sigma_{i}^{c}$ to obtain $\alpha\left(\Sigma_{b}, \gamma\right)$.

In the case $\Gamma_{i} \gg \Gamma_{b}$, debonding is impossible, $\beta=0$, and $U$ becomes a function of $\alpha$ alone. Minimisation of $\mathcal{E}_{\text {tot }}$ (Eq. 1) then gives $\Sigma_{b}^{c}(\alpha)=\left[-\frac{\mathrm{d}(\alpha U(\alpha))}{\mathrm{d} \alpha}\right]^{-1 / 2}$. For small $\alpha$ (large segments), the cracks do not interact with each other; the size of the zone where the elastic energy is released (in white on Fig. 2b) is independent of the distance between cracks [28]; so that $\Sigma_{b}^{c}(\alpha) \simeq \Sigma_{b}^{0}$ is constant, the crack density remains undetermined and an additional selection criteria has to be invoked to determine the spacing. For large $\alpha$, the stresses are released everywhere except in the vicinity of the substrate [28], thus the elastic energy is stored in a surface (in red on Fig. 2c) of typical size $\frac{1}{\alpha^{2}}$, so that $U \propto \frac{1}{\alpha^{2}}$ and $\Sigma_{b}^{c}(\alpha)=\Delta \Sigma_{b}^{c} \alpha$ is proportional to $\alpha$. Using the Finite Element Method (FEM) to solve the 2D elasticity problems for different segment aspect ratio $\alpha$, we obtain an accurate approximation of $\Sigma_{b}^{c}(\alpha)$ (Fig. 2 for $\nu=0.3$ and [28] for other values of $\nu)$. This allows us to highlight that the crack interaction surprisingly becomes effective as soon as $\alpha \simeq 0.1$ and the linear behaviour, as soon as $\alpha \simeq 0.4$, that is for quite small values of the channeling crack density $\alpha$.

Now, we consider a given $\alpha$ and look for the loading $\Sigma_{i}^{c}$ corresponding to the debonding. First, we determine by FEM, the critical loading parameter $\Sigma_{i}^{c}(\alpha, \beta)$ which minimises (1) at fixed $\alpha$ for different debonding density $\beta$. We get $\Sigma_{i}^{c}(\alpha, \beta)=\left[-\alpha \frac{\partial(U(\alpha, \beta))}{\partial \beta}\right]^{-1 / 2}$. For increasing $\beta$, $\Sigma_{i}^{c}$ quickly decreases from infinity down to a minimum and than slowly increases (inset Fig. of 3 ). Since $\Sigma_{i}^{c} \rightarrow_{\beta \rightarrow 0}$ $\infty$ [44], the onset of debonding is impossible without the presence of a defect. But, the minimum of $\Sigma_{i}^{c}$ is reached for $\beta \ll 1$ so that in practice a small defect is able to initiate the debonding. The further slow increase makes the initiation loading weakly sensitive to the size of the defects. Also, one can safely define the onset of debonding by $\Sigma_{i}^{c}(\alpha) \equiv \min _{\beta} \Sigma_{i}^{c}(\alpha, \beta)$. We get $\Sigma_{i}^{c}(\alpha) \simeq \Sigma_{i}^{0}$ constant for $\alpha \lesssim 0.1$ and $\Sigma_{i}^{c} \simeq \Delta \Sigma_{i}^{c} \sqrt{\alpha}$ for $\alpha \gtrsim 0.4$ (Fig. 3 for $\nu=0.3$ and [28] for other values of $\nu$ ). This behaviour can

\footnotetext{
${ }^{3}$ This makes our results not directly applicable to cases where debonding occurs at the same time than channeling, as e.g. to [42, $43]$.
}

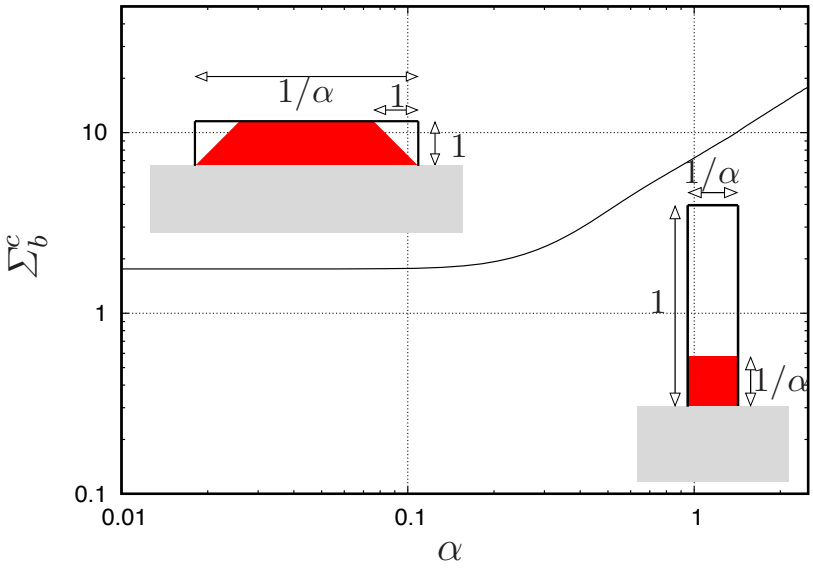

Fig. 2: In the absence of debonding, that is for $\Gamma_{i} \gg \Gamma_{b}$, the channeling crack density $\alpha=h / \ell$ increases with $\Sigma_{b}^{c}$, that is when the loading $\sigma_{0}$, the thickness $h$ or the brittleness $\left(\Gamma_{b} E\right)^{-1}$ of the layer increases (here, $\nu=0.3$ ). The sketches (b) and (c) represent one segment in the case of small or large $\alpha$ respectively; the surface in red corresponds approximatively to constraint zones, whereas the stresses are relieved by the free boundaries in the white zones (see also Fig. 2 in suppl. mat. [28]). (b) For small values of $\alpha(\alpha \lesssim 0.1)$, the released zones are independent that is, the cracks do not interact mutually, so that $\Sigma_{b}^{c} \simeq \Sigma_{b}^{0}$ is constant. (c) For larger values of $\alpha(\alpha \gtrsim 0.4)$, $\Sigma_{b}^{c} \simeq \Delta \Sigma_{b}^{c} \alpha$; this can be deduced from the fact that the energy is released in a zone scaling with $\frac{1}{\alpha}\left(1-\frac{1}{\alpha}\right)$.

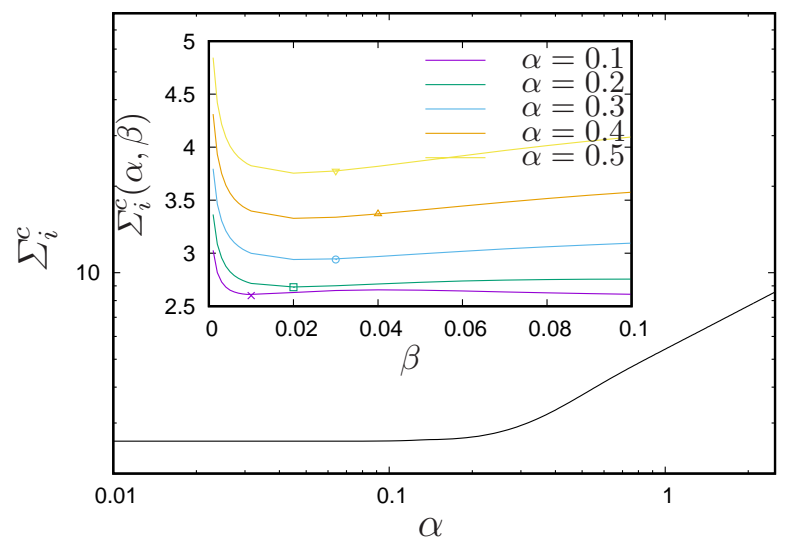

Fig. 3: Debonding loading $\Sigma_{i}^{c}$ for different channeling $(\alpha)$ and debonding $(\beta)$ densities (case $\nu=0.3$ ). Inset: evolution of $\Sigma_{i}^{c}(\beta, \alpha)$ as a function of $\beta$ for a given value $\alpha . \Sigma_{i}^{c}(\beta, \alpha)$ reaches a minimum for $\beta \ll 1$, that we use to define the onset of debonding $\Sigma_{i}^{c}(\alpha)$ plotted in the main frame. For small crack density, $\Sigma_{i}^{c} \simeq \Sigma_{i}^{0}$ is constant. For larger values of $\alpha, \Sigma_{i}^{c} \simeq$ $\Delta \Sigma_{i}^{c} \sqrt{\alpha}$.

be corroborated as follows. For small $\alpha, \Sigma_{i}^{c}(\alpha)$ is constant, since each channeling crack behaves independently. For larger $\alpha$, debonding over a small length $\frac{\beta}{\alpha}$ releases energy in the red zone of Fig. 2c over a surface proportional to $\frac{\beta}{\alpha} \frac{1}{\alpha}$ [28]; so that $U \propto\left(\frac{1}{\alpha}-\frac{\beta}{\alpha}\right) \frac{1}{\alpha}$, which gives $\Sigma_{i}^{c}(\alpha)=\Delta \Sigma_{i}^{c} \sqrt{\alpha}$ proportional to $\sqrt{\alpha}$.

It is now time to compare $\Sigma_{b}$ with the loading needed for channeling $\Sigma_{b}^{c}$, and the one needed for debonding $\sqrt{\gamma} \Sigma_{i}^{c}$. 
For $\gamma<\left(\frac{\Sigma_{b}^{0}}{\Sigma_{i}^{0}}\right)^{2}$ (inset of Fig. 4$), \sqrt{\gamma} \Sigma_{i}^{c}(\alpha)<\Sigma_{b}^{c}$ whatever the value of $\alpha$ so that debonding is favoured against channeling and occurs as soon as $\Sigma_{i}=\Sigma_{i}^{0}$. For $\gamma>\left(\frac{\Sigma_{b}^{0}}{\Sigma_{i}^{0}}\right)^{2}$ (case considered until the end of the paper), $\Sigma_{b}^{c}(\alpha)$ and $\sqrt{\gamma} \Sigma_{i}^{c}(\alpha)$ intersect at a point $\left(\Sigma_{b}^{d}, \alpha_{c}\right)$ depending on the value $\gamma$ alone (Fig. 4 and Fig. 5 of [28]). We find that if $\gamma \gtrsim 0.5$, approximate formulae for the intersection point are $\alpha_{c} \simeq \frac{\gamma}{2}$ and $\Sigma_{b}^{d} / \Sigma_{b}^{0} \simeq 2 \gamma$ whatever the value of $\nu$ and in both plane strain and stress hypotheses [28].

When the loading parameter $\Sigma_{b}$ increases, the following scenario occurs, assuming as discussed previously that the global minimum is reached after the nucleation and propagation phases of the channeling cracks (Fig. 4):

1. for $\Sigma_{b} \leq \Sigma_{b}^{0}$, the available elastic energy is too small for crack to channel through the layer. Below this value some star-shaped cracks may nucleate locally and propagate following a jerky path ruled by local inhomogeneities [1], but the available elastic energy is to weak to obtain a straight propagation through the layer.

2. for $\Sigma_{b}^{0}<\Sigma_{b} \leq \Sigma_{b}^{d}$, channeling without debonding occurs, since $\Sigma_{b}^{c}(\alpha)<\sqrt{\gamma} \Sigma_{i}^{c}(\alpha)$ in this zone. As long as the cracks do not interact with each others, they appear at arbitrary locations corresponding to weaker heterogeneities. Once the cracks are close enough ( $\alpha \simeq 0.1$ ), the distance between them becomes deterministic and is ruled by elastic interactions [27].

3. for $\Sigma_{b}>\Sigma_{b}^{d}$, no subsequent channeling crack appears and the additional bulk energy is dissipated in debonding. Indeed the loading needed for debonding $\left(\Sigma_{i}^{c}\left(\alpha_{c}, \beta\right)\right)$ increases slowly (Fig. 3inset) with $\beta$ and stays smaller than the one $\left(\Sigma_{b}^{c}(\alpha), \alpha>\alpha_{c}\right)$ needed to create additional channel cracks. Hence $\alpha$ saturates at $\alpha_{c}$.

Thus, simply by balancing the elastic energy and channeling fracture costs with an additional dissipation source (here debonding), it is possible to understand the increase of $\alpha$ with the loading until saturation observed in several experiments $[16,39]$.

Let us now assume that the cracks are formed under increasing loading and discuss the influence of the thickness on the final pattern for a given maximum loading $\sigma_{0}^{\max }$ and a given material characterized by its fracture toughness $K_{c} \equiv \sqrt{\frac{\Gamma_{b} E}{1-\nu^{2}}}$. The boundaries $\Sigma_{b}^{c}$ and $\Sigma_{b}^{d}$ defines two critical thicknesses: First, $h_{c}=\Gamma_{b} E\left(\frac{\sum_{b}^{0}}{\sigma_{0}^{\max }}\right)^{2}=$ $K_{c}^{2}\left(1-\nu^{2}\right)\left(\frac{\Sigma_{b}^{0}}{\sigma_{0}^{\max }}\right)^{2}$ below which no channeling cracks can propagate before the maximum loading $\sigma_{0}^{\max }$ is reached and second, $h_{d}=\Gamma_{b} E\left(\frac{\sum_{b}^{d}(\gamma)}{\sigma_{0}^{\max }}\right)^{2}=h_{c}\left(\frac{\sum_{b}^{d}(\gamma)}{\sigma_{0}^{\max }}\right)^{2} \sim 4 \gamma^{2} h_{c}$

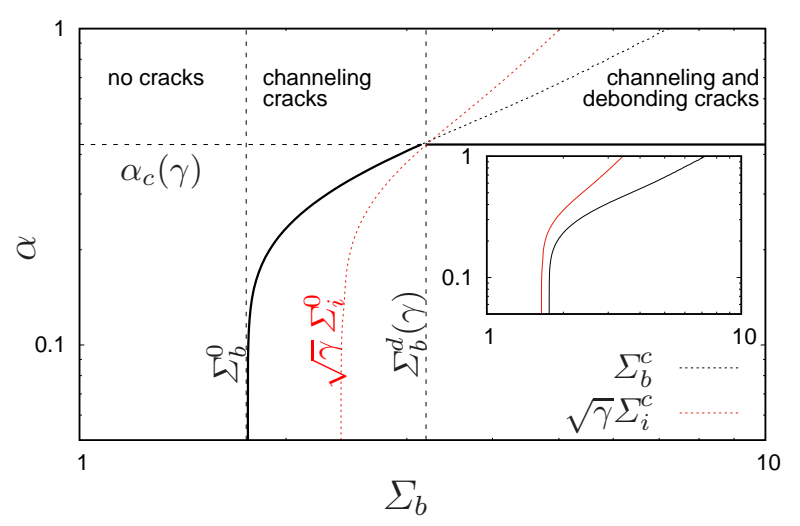

Fig. 4: Evolution of the crack density $\alpha$ with increasing loading parameter $\Sigma_{b}(\nu=0.3)$. In the case $\sqrt{\gamma} \Sigma_{i}^{0}<\Sigma_{b}^{0}$ (inset where $\gamma=0.4), \sqrt{\gamma} \Sigma_{i}^{c}<\Sigma_{b}^{c}$, so that debonding is favoured over channeling as soon as $\Sigma_{b}>\sqrt{\gamma} \Sigma_{i}^{c}$. In the case $\sqrt{\gamma} \Sigma_{i}^{0}>\Sigma_{b}^{0}$ $\left(\gamma \equiv \Gamma_{i} / \Gamma_{b}=0.86\right.$ in the main plot), the curves $\Sigma_{b}^{c}$ and $\sqrt{\gamma} \Sigma_{i}^{c}$ intersect at a point $\left(\alpha_{c}, \Sigma_{b}^{d}\right)(\gamma)$. As long as $\Sigma_{b}<\Sigma_{b}^{0}$, no cracks can propagate. For $\Sigma_{b}^{0}<\Sigma_{b}<\Sigma_{b}^{d}(\gamma)$, channeling cracks appear progressively and follow the evolution of $\Sigma_{b}^{c}(\alpha)$ (corresponding to Fig. 2): $\alpha$ is independent from $\Sigma_{b}$ in the diluted regime $(\alpha \lesssim 0.1)$ and increases linearly with $\Sigma_{b}$ once $\alpha \gtrsim 0.4$. For $\Sigma_{b}=\widetilde{\Sigma}_{b}^{d}(\gamma), \Sigma_{b}^{c}(\alpha)$ intersects the debonding onset loading $\gamma \Sigma_{i}^{c}(\alpha)$ (red dashed plot taken from Fig. 3). Above this value $\left(\Sigma_{b}>\Sigma_{b}^{d}(\gamma)\right)$, no additional channeling crack appears and the available elastic energy is transferred to debonding, so that $\alpha$ saturates at $\alpha_{c}(\gamma)$.

below which no debonding can occur before $\sigma_{0}^{\max }$. From the knowledge of $h_{c}$ and $h_{d}$, one can thus easily obtain the toughness $K_{c}$ (if $\sigma_{0}$ is known) and $\Sigma_{b}^{d}(\gamma)$, that is $\gamma$. Also, Fig. 4 can be plotted under the form $\ell / h_{c}$ as a function of $h / h_{c}$ (black line in Fig. 5) since $\ell / h_{c}=(1 / \alpha)\left(h / h_{c}\right)$ and $h / h_{c}=\left(\Sigma_{b} / \Sigma_{b}^{0}\right)^{2}$ (use Eq. (2) and definition of $\left.h_{c}\right)$. Contrary to Fig. 4, this curve is nearly independent of $\nu$ and is valid for both plane stress and strain hypotheses [28]. For $h_{c}<h<h_{d}$, channeling occurs without debonding. The final spacing $\ell$ decreases with $h$ in a tiny zone, then the square root dependance of $\ell$ with $h$ is retrieved; a good approximation is $\ell \simeq 4 \sqrt{h h_{c}}$ for $\frac{h}{h_{c}} \gtrsim 2.5$. For $h>h_{d}$, channeling has stopped once debonding has occurred, this fixes the final crack density, so that the spacing $\ell$ evolves linearly with $h$; a good approximation is $\ell \simeq 2 h / \gamma$. In $3 \mathrm{D}$, pattern formation becomes more complex, but similar scalings are awaited with slightly different pre-factors [28].

The influence of the thickness on the final crack pattern (Fig. 1a) formed during the drying of a hard nano-latex (radius $a \simeq 15 \mathrm{~nm}$ ) colloidal suspension has been investigated experimentally in [1]. A layer of liquid dispersion is dried until the particles form a brittle solid porous material saturated with water. The solid then shrinks as the liquid evaporates due to the progressive build up of an isotropic prestress $\sigma_{0}$ in the layer. At the particle scale, $\sigma_{0}$ is linked to the capillary pressure due to the meniscus formed between the nano-particles at the air/liquid interface. The prestress grows following the theory of 
poro-elasticity [29], until the radius of curvature of the meniscus becomes maximum [39]. For a dense packing of spherical particules, this corresponds to $\sigma_{0}^{\max } \simeq 5.3 \gamma_{0} / a$ [45]. Since the contraction is constrained by the adhesion to the substrate, tensile stresses progressively develop that possibly give rise to cracks. The three final patterns predicted by the model are retrieved depending on the layer thickness: (i) for $h<h_{c}$, some small star-shape nucleation cracks or some jerky paths may be present, but no straight channel crack crossing the layer since the available elastic energy was not sufficient to propagate any crack across the whole layer; (ii) for $h_{c}<h<h_{d}$, the layer is cut into polygons by channeling cracks that appear hierarchically [46], but debonding does not occur; (iii) for $h_{d}<h$, progressive division of the layer in polygones is followed by progressive debonding. The critical thicknesses are approximately given by $h_{c} \sim 10 \mu \mathrm{m}$ and $h_{d} \sim 30 \mu \mathrm{m}$. This gives, inverting the model and taking $\sigma_{0}^{\max } \simeq 5.3 \gamma_{0} / a \simeq 25 \mathrm{MPa}\left(\gamma_{0}=0.07 \mathrm{~N} / \mathrm{m}\right.$ is the surface tension of water), $K_{c} \simeq 0.02-0.06 \mathrm{MPa}^{1 / 2}$ for $\nu=0.2-0.4$ and $\gamma \sim 0.9$ which sounds reasonable in regard of more conventional materials [47]. Remarkably, the method provides an estimate of $K_{c}$ at the moment of fracture, that is before the end of the consolidation, value which is inaccessible by more conventional methods based on tensile fracture tests. The behaviour of the final spacing $\ell$ with $h$, reported on Fig. 5 , is in qualitative agreement with the theoretical solution. The underestimation of $\ell / h_{c}$ by the model may be explained by the omission of other than fracture and bulk energy consumptions (e.g. elastic deformation of the substrate, friction or plasticity [48]). Other hypotheses made here are also questionable, notably (i) the omission of the crack propagation history and the hypothesis that the final pattern corresponds to a global minimum state of the total energy and (ii) the 2D simplified geometry of the effective 3D crack pattern [28].

In previous drying experiments, measurements made in the regime without debonding (for $h_{c}<h<h_{d}$ ) are insufficient to make any comparison. Therefore, the spacing obtained for some epitaxial films [49] is added on Fig. 5. The square root scaling is in agreement with the channeling regime, suggesting the absence of debonding (this indication is however lacking in the paper). The quantitative discrepancy may be explained by the elasticity of the substrate which increases the spacing [50] since it absorbes some elastic energy; But this elasticity is not taken into account in our solution contrary to theirs. Other papers $[13,51,52]$ report a constant value of $\alpha$ independently of $h$, decreasing when the adhesion decreases, apparently in qualitative agreement with our model. It is however difficult to conclude since adhesion may play on both, debonding energy and bottom friction (which changes the boundary condition at the substrate hence the stress field and elastic energies). Generally speaking, it seems difficult to fully test this approach and notably the key role (highlighted here) of the adhesion strength, using the numerous measurements of crack spacing available in the literature.

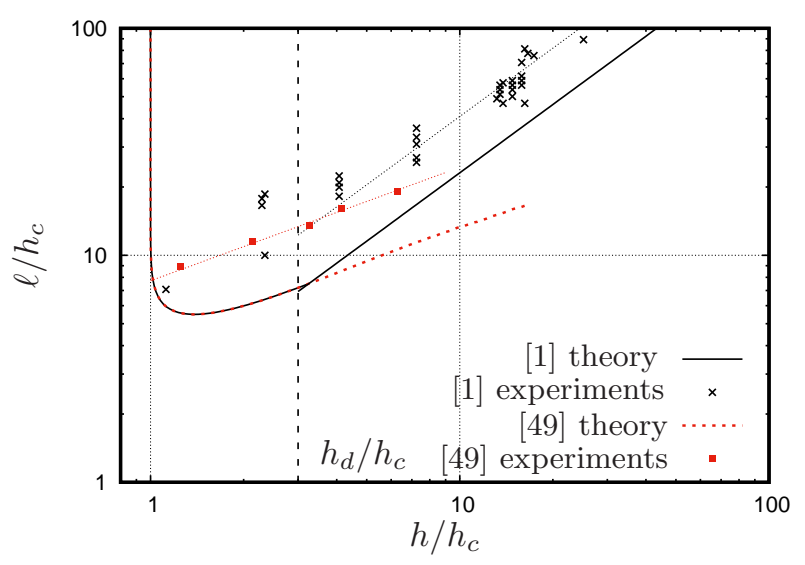

Fig. 5: Evolution of the crack spacing $\ell$ with the film thickness $h$, deduced from Fig. 4 . The nondimensionalization by $h_{c}$ renders this plot independent of $\nu$. For $h<h_{c}$, neither channeling nor debonding cracks have enough energy to propagate. For $h_{c}<h<h_{d}, \ell \propto \sqrt{h}$; this square-root scaling is in agreement with the one observed in epitaxial films [49]. For $h>h_{d}, \ell \propto h$ in agreement with drying of colloid experiments [1]. In those experiments, $h_{c} \sim 10 \mu \mathrm{m}$ and $h_{d} \sim 30 \mu \mathrm{m}$, yielding $\gamma \simeq 0.86$ corresponding to this plot; the crossover from the square-root to the linear regime at $h_{d} / h_{c}$ is barely apparent (Dashed line on experimental points are guides for the eyes). Several reasons may explain that our model underestimates the spacing: the omission of some other sources of energy (e.g. elastic deformation of the substrate, friction or plasticity); the simplified $2 \mathrm{D}$ geometry; the omission of the crack propagation history and the correlated hypothesis that the final pattern corresponds to a global minimum of the energy.

\section{Performing new experiments is necessary to this end.}

To summarize, the cracks interact mutually as soon as their spacing becomes smaller than ten times the thickness of the layer. Then, balancing elastic with fracture bulk and interface energies provides the channeling crack density in a tensile brittle fracture layer as a function of two dimensionless parameters: $\Sigma_{b}$ which provides the ratio between the elastic energy stored in the layer and the fracture energy, and $\gamma$ which is given by the ratio between the layer fracture energy and the adhesion energy at the layer/substrate interface. These two dimensionless parameters are sufficient to understand a broad number of observations going from the square-root or linear increase of the spacing with the thickness, to its decrease with loading until saturation; the adhesion strength being identified as a key ingredient of these behaviour changes. We illustrate on an example how readily measurable quantities, as the critical onset of channeling and debonding thicknesses, can be used to characterize the material and the interface strength. Along a similar line, it would also be possible to inverse the model to obtain some clues on the loading at the origin of natural crack patterns on Earth or other planets. Finally, the present modelling framework is versatile, its extension to other geometries (e.g. multilayers [38], cylinder [52]), loading (e.g. uniaxial tension of the substrate [16]) and dissipation sources (e.g. plasticity [12], 
friction [53], spalling [15], which may also yield saturation of the crack density with the loading), is quite straightforward, broadening the range of applicability, whether in geosciences or engineering.

\section{$* * *$}

This work has benefited from Institut Universitaire de France. I thank L. Pauchard for giving me the chance to discover the topic of colloidal drying, for the nice experiments [1] and for the picture of Fig. 1; I am also grateful to D. Bonamy, A. Davaille, E. Dressaire, G. Gauthier, S. Paillat, N. Ribe for helpful discussions and encouragements. I thank the anonymous referees for their relevant remarks and suggestions that have given me the opportunity to improve the manuscript.

\section{REFERENCES}

[1] Lazarus V. and Pauchard L., Soft Matter, 7 (2011) 2552.

[2] Goehring L., Nakahara A., Dutta T., Kitsunezaki S. and TARAFDAR S., Desiccation cracks and their patterns: Formation and modelling in Science and Nature (Wiley) 2015.

[3] Pauchard L., Lazarus V., Abou B., Sekimoto K., Aitken G. and Lahanier C., Reflets de la Physique, Société Française de Physique, 3 (2007) 5 https://hal.archives-ouvertes.fr/hal-00158845.

[4] Aydin A. and Pollard D. D., Geological Society of America Bulletin, 100 (1988) 1181.

[5] Goenring L., Philosophical Transactions of the Royal Society of London A: Mathematical, Physical and Engineering Sciences, 371 (2013) 20120353.

[6] Smrekar S. E., Moreels P. and Franklin B. J., Journal of Geophysical Research: Planets, 107 (2002) 5098.

[7] Levy J., Head J. and Marchant D., J. Geophys. Res., 114 (2009) E01007.

[8] Freund L. B. and Suresh S., Thin film materials; stress, defect formation and surface evolution (Cambridge University press, Cambridge, UK) 2004.

[9] Keddie J. and Routh A. F., Fundamentals of Latex Film Formation (Springer) 2010.

[10] Evans A. G., Mumm D. R., Hutchinson J. W., Meier G. H. and Pettit F. S., Progress in Materials Science, 46 (2001) 505.

[11] Seghir R. and Arscott S., Scientific Reports, 5 (2015) 14787.

[12] Nagl M. M., Saunders S. R. J., Evans W. T. and Hall D. J., Corrosion Science, 35 (1993) 965.

[13] Groisman A. and Kaplan E., Europhysics Letters, 25 (1994) 415.

[14] Colina H. and Roux S., European Physical Journal E, 1 (2000) 189.

[15] Leterrier Y., Waller J., MÅnson J. A. E. and NAirn J. A., Mechanics of Materials, 42 (2010) 326.

[16] Wu H. Q. and Pollard D. D., J. Struct. Geology, 17 (1995) 887.

[17] Bai T. X. and Pollard D. D., J. Struct. Geology, 22 (2000) 43.
[18] Kappert E. J., Pavlenko D., Malzbender J., NijmeiJer A., Benes N. E. and Tsai P. A., Soft Matter, 11 (2015) 882.

[19] Lachenbruch A., J. Geophysical Research, 66 (1961) 4273.

[20] Bai T. X., Pollard D. D. and Gao H. J., Nature, 403 (2000) 753.

[21] Adda-Bedia M. and Ben Amar M., Physical Review Letters, 86 (2001) 5703.

[22] Yin H. M., International Journal of Solids and Structures, 47 (2010) 1007.

[23] BaO C., TANG C. A., CAi M. and TANG S., International Journal of Fracture, 181 (2013) 241.

[24] Bai T. X., Pollard D. D. and Gao H. J., International Journal of Fracture, 103 (2000) 373.

[25] Lawn B., Fracture of brittle solids-Second edition (The Press Syndicate of the University of Cambridge, Cambridge, UK) 1993.

[26] Beuth J. L., International Journal of Solids and Structures, 29 (1992) 1657.

[27] Bohn S., Platkiewicz J., Andreotti B., Adda-Bedia M. and Couder Y., Physical Review E, 71 (2005) 046215.

[28] Supplemental material: details on how the solution is obtained; solutions for other values of $\nu$ and plane stress hypotheses; extension to $3 d$.

[29] Chekchaki M. and Lazarus V., Transport in Porous Media, 100 (2013) 143.

[30] Hutchinson J. W. and Suo Z., Mixed Mode Cracking in Layered Materials Vol. 29 (Academic Press) 1992 pp. 63-191.

[31] Hutchinson J. W. and Xia Z., Journal of the Mechanics and Physics of Solids, 48 (2000) 1107.

[32] Griffith A. A., Philosophical Transactions of the Royal Society of London, 221 (1920) 163.

[33] Francfort G. A. and Marigo J. J., Journal of the Mechanics and Physics of Solids, 46 (1998) 1319.

[34] Gauthier G., Lazarus V. and Pauchard L., EPL, 89 (2010) 26002.

[35] Maurini C., Bourdin B., Gauthier G. and Lazarus V., International Journal of Fracture, 184 (2013) 75.

[36] Vandenberghe N., Vermorel R. and Villermaux E., Physical Review Letters, 110 (2013) 174302.

[37] Bourdin B., Marigo J.-J., Maurini C. and Sicsic P., Physical Review Letters, 112 (2014) 014301.

[38] Leung D., He M. and Evans A., Journal of Materials Research, 10 (1995) 1693.

[39] Man W. and Russel W. B., Physical Review Letters, 100 (2008) 198302.

[40] Baldelli A. A. L., Bourdin B., Marigo J.-J. and Maurini C., Continuum Mechanics and Thermodynamics, 25 (2013) 243.

[41] León Baldelli A. A., Babadjian J. F., Bourdin B., Henao D. and Maurini C., Journal of the Mechanics and Physics of Solids, 70 (2014) 320.

[42] Allain C. and Limat L., Physical Review Letters, 74 (1995) 2981.

[43] Marthelot J., Roman B., Bico J., Teisseire J., Dalmas D. and Melo F., Physical Review Letters, 113 (2014) 085502.

[44] Yu H. H. and Hutchinson J. W., Thin Solid Films, 423 (2003) 54.

[45] Mason G. and Mellor D., Journal of colloid and inter- 
face science, 176 (1995) 214.

[46] Bohn S., Pauchard L. and Couder Y., Physical Review E, 71 (2005) 046214.

[47] Ashby M., Acta Metallurgica, 37 (1989) 1273.

[48] Goenring L., Clegg W. J. and Routh A. F., Physical Review Letters110, 2013 () .

[49] Thouless M. D., Olsson E. and Gupta A., Acta Metallurgica Et Materialia, 40 (1992) 1287.

[50] Shenoy V. B., Schwartzman A. F. and Freund L. B., International Journal of Fracture, 109 (2001) 29.

[51] Shorlin K. A., de Bruyn J. R., Graham M. and MorRIS S. W., Physical Review E, 61 (2000) 6950.

[52] Boulogne F., Pauchard L. and Giorgiutti-Dauphiné F., EPL (Europhysics Letters), 102 (2013) 39002.

[53] Schöpfer M. P. J., Arslan A., Walsh J. J. and Childs C., Journal of Structural Geology, 33 (2011) 551. 